\title{
DRL performance in rats with medial or lateral septal lesions
}

\author{
S. BROOKES, J. N. P. RAWLINS, J. A. GRAY, and J. FELDON \\ University of Oxford, Oxford, England
}

\begin{abstract}
Electrolytic lesions restricted to either the medial or the dorsolateral septal area were made in rats previously implanted with chronic recording electrodes in the hippocampal formation. The former, but not the latter, lesion disrupted hippocampal theta activity. Rats with both kinds of lesion, as well as operated controls, were trained to barpress for food pellets on a schedule of differential reinforcement of low rates with an interresponse requirement of 20 sec. Both lesions impaired task efficiency to an equal degree, but neither prevented the development of a timing curve. This pattern of results is consistent with the behavioral inhibition hypothesis of septal function, but the failure to dissociate the effects of the two lesions is discordant with observations in other tasks apparently requiring response inhibition.
\end{abstract}

The close anatomical and physiological relationships between the septal area and the hippocampus are well known (Elliott \& Whelan, 1978). The medial septal area is the origin of a major cholinergic projection to the hippocampal formation; the lateral septal area, in contrast, is the target of the main hippocampal subcortical projection (Swanson, 1978). A projection from the lateral to the medial septal area completes the circuit (Krayniak, Weiner, \& Siegel, 1980; Swanson \& Cowan, 1979). A further difference between the medial and lateral septal areas lies in their relationship to the hippocampal theta rhythm (high-voltage, rhythmic slow waves in the 6-12-Hz range). The theta rhythm depends on pacemaker cells located in the medial septal area and does not survive destruction of this region; it persists, however, after damage confined to the lateral septal area (Petsche, Stumpf, \& Gogolak, 1962; Rawlins, Feldon, \& Gray, 1979).

We have previously reported a double dissociation between the behavioral effects of medial and lateral septal lesions. In those experiments, Feldon and Gray (1979a, 1979b) measured the resistance to extinction of a running response after food reward had been delivered during acquisition on either a continuous or a partial reinforcement schedule. Medial, but not lateral, septal lesions increased resistance to extinction regardless of the acquisition schedule; lateral, but not medial, septal lesions abolished the partial reinforcement extinction effect, that is, the increase

This research was supported by the U.K. Medical Research Council. J.N.P.R. is Henry Head Research Fellow of the Royal Society and Senior Research Fellow at University College, Oxford. J.F. held the Kenneth Lindsay Scholarship from the Anglo-Israel Association; he is now at the Department of Psychology, Tel Aviv University, Israel. We thank Neil McNaughton and Cliff Preston for statistical advice. Requests for reprints should be sent to J. A. Gray, Department of Experimental Psychology, University of Oxford, South Parks Road, Oxford OX1 3UD, England. in resistance to extinction that is normally produced by partial, relative to continuous, reinforcement. Total septal (Feldon \& Gray, 1979a; Henke, 1974, 1977) and total hippocampal (Rawlins, Feldon, \& Gray, 1980) lesions produce a combination of the separate changes seen after medial and lateral septal lesions, respectively: increased resistance to extinction in the continuous reinforcement condition plus abolition of the partial reinforcement extinction effect. This pattern of change is also seen after complete section of the fornix-fimbria (Feldon, Rawlins, \& Gray, Note 1), although not after section of the fimbria alone (Rawlins et al., 1980). Thus, one may reasonably attribute the separate effects of medial and lateral septal lesions on resistance to extinction to the fact that the former lesion de-afferents the hippocampus while the latter de-efferents it (Gray, Feldon, Rawlins, Owen, \& McNaughton, 1978).

Resistance to extinction presumably reflects an animal's response to the omission of reward encountered by both continuously and partially reinforced subjects during extinction but only partially reinforced subjects during acquisition. Another task that involves repeated failure to obtain reward for instrumental responding is that of performing on a schedule of differential reinforcement of low rates of response (DRL) in the Skinner box. It has been repeatedly reported that such performance is impaired after both large septal (e.g., Ellen, Wilson, \& Powell, 1964) and large hippocampal (e.g., Pellegrino \& Clapp, 1971) lesions; for a review, see Gray and McNaughton (1983). On a DRL schedule, as during simple extinction, animals normally come to respond more and more slowly as fast responses go unrewarded. If DRL is analogous to extinction in this way, then, on the basis of Feldon and Gray's (1979a, 1979b) findings, one might predict that medial septal damage (which increases resistance to ex- 
tinction) is the critical component of a large septal lesion in causing the deficit in DRL performance. Since Feldon and Gray (1979a, 1979b) did not find increased resistance to extinction after lateral septal lesions, then, by the same argument, septal damage restricted to this area should not impair DRL performance.

One may also compare DRL performance to training on a partial reinforcement schedule. In both cases, the animal's final level of performance is presumably determined in part by the balance between the inhibitory effects of nonreward and the excitatory effects of reward (cf. Amsel's, 1962, analysis of the effects of partial reinforcement). One factor that contributes to the eventual determination of the balance point is the process described by Amsel (1962) as "counterconditioning." That is to say, on a partial reinforcement schedule, the inhibitory effects of nonreward gradually become less as nonreward becomes a cue for the continued performance of the sometimes-rewarded response. The conditions for a similar process exist also on a DRL schedule, although we know of no behavioral analysis of the role it might play. In the partial reinforcement task, both total (Henke, 1974) and lateral (Feldon \& Gray, 1979a, 1979b) septal lesions appear to weaken the process of counterconditioning (Gray et al., 1978). If the same thing were to occur in animals performing on a DRL schedule after lateral septal lesions, one might expect a shift (relative to intact animals) towards lower response rates; other things being equal, this would show up as an improved DRL efficiency. At the least, given Feldon and Gray's (1979a, 1979b) findings, we would not expect animals with lateral septal lesions to show the impairment in DRL performance that is characteristic of animals with total septal lesions.

These arguments converge, then, on the two predictions tested in the experiment reported here: (1) that medial septal lesions would reproduce the DRL deficit found in animals with total septal lesions, and (2) that lateral septal lesions would either leave DRL performance unchanged or improve it. The first of these predictions can also be derived from a number of more general arguments which attribute the communality between the behavioral effects of septal and hippocampal lesions to the abolition of the hippocampal theta rhythm produced by septal lesions (e.g., Donovick, 1968; Gray, 1970; O’Keefe \& Nadel, 1978).

Previous reports are ambiguous with respect to these predictions. Burkett and Bunnell (1966) and Ellen et al. (1964) reported data consistent with a medial septal focus for the DRL deficit. Agnew and Mayer (1969), on the other hand, found a deficit after both medial and lateral septal lesions and no difference between the two. However, in none of these experiments were explicit, functionally derived criteria used to define medial and lateral septal damage. In the present experiment, as in others from this laboratory (Feldon \& Gray, 1979a, 1979b, 1981; Feldon, Rawlins, \& Gray, 1982a, 1982b), the histological evaluation of the lesion was based upon previously established electrophysiological criteria (Rawlins, Feldon, \& Gray, 1979). These criteria consist in the extent of loss in the amplitude of the hippocampal theta rhythm caused by the lesion: a medial septal lesion should cause a considerable loss, a lateral septal lesion only minimal loss (Rawlins et al., 1979). In the experiment reported here, the theta rhythm was recorded before and after the lesion in all animals; subsequent allocation to the medial or lateral septal condition was thus based upon both electrophysiological and electrophysiologically derived histological criteria.

\section{METHOD}

\section{Subjects}

The subjects were 29 naive male Sprague-Dawley rats (375$608 \mathrm{~g}$ ), supplied by Oxfordshire Laboratory Animal Colonies. Following arrival and before use in the experiments, they were allowed at least 2 weeks ad-lib food and water. Two weeks before the beginning of behavioral training, they were gradually introduced to a food-deprivation schedule, allowing free access to food for $1 \mathrm{~h}$ per day. This schedule was maintained throughout the experiment. Feeding took place at least $1 \mathrm{~h}$ after the last animal was run each day. The animals were caged singly, with ad-lib water. During the 80-day course of the behavioral experiment, all the rats increased in body weight: the controls by $9.7 \%$, the medial septal lesion group by $11.5 \%$, and the lateral septal lesion group by $17.4 \%$. Details of the composition of the experimental groups are given below.

\section{Surgery and Histology}

All procedures have been fully described by Rawlins et al. (1979). All subjects were implanted under Equithesin anesthesia $(3 \mathrm{ml} / \mathrm{kg}$ ) with two bipolar recording electrodes (Diamel-insulated stainless steel wire, $150 \mu$ in diameter, cut square and twisted to leave $2-\mathrm{mm}$ vertical separation between the tips) bilaterally in the dorsomedial subiculum (James, McNaughton, Rawlins, Feldon, \& Gray, 1977); coordinates, with skull flat between bregma and lambda, were: $6 \mathrm{~mm}$ posterior to bregma and $2 \mathrm{~mm}$ lateral, and lower tip $4 \mathrm{~mm}$ deep, from dura. The subjects intended for medial septal lesions were, in addition, implanted with a monopolar lesioning electrode (250- $\mu$ diameter; $1 \mathrm{~mm}$ anterior, directly on the midline and $5 \mathrm{~mm}$ deep). The subjects intended for lateral septal lesions were implanted with two monopolar lesioning electrodes (250- $\mu$ diameter; $0.5 \mathrm{~mm}$ anterior, $\pm 0.7 \mathrm{~mm}$ lateral, $4.5 \mathrm{~mm}$ deep). All electrodes were led out to an Amphenol plug and secured to the skull with dental acrylic and stainless steel screws; a silver wire wrapped around the screws served as ground. Lesions (made later; see below) were carried out under ether anesthesia by passing anodal direct current through the lesioning electrodes; crocodile clips on the ears served as the cathode. Current was $1 \mathrm{~mA}$ for $15 \mathrm{sec}$ for the medial septal lesion, and $0.5 \mathrm{~mA}$ for $20 \mathrm{sec}$ via each electrode in turn for the lateral septal lesion.

At the end of the experiment, the subjects were killed with an overdose of pentobarbitone and perfused through the heart with isotonic saline and $10 \%$ Formalin. The brains were left in formol saline for at least 2 weeks before being embedded in celloidin. They were sectioned at $30 \mu$, and every fifth section was stained with cresyl violet. The lesions were reconstructed by being drawn on plates derived from König and Klippel (1963). Histological 
criteria for acceptable lesions were as previously described (Feldon \& Gray, 1979a, 1979b; Feldon, Rawlins, \& Gray, 1982; Rawlins et al., 1979).

\section{Electrophysiology and Allocation to Groups}

Recording was accomplished on a Grass 79D polygraph, to which the rat was connected via shielded cable; movement artifact was eliminated by a source-follower attached to the cable close to the connection with the animal's plug. The recording conditions and methods for assessing the degree of loss of hippocampal theta after lesioning were as described by Rawlins et al. (1979). A prelesion record was taken from the recording electrode implanted in each hemisphere immediately before current was passed; at least 2 weeks elapsed before the postlesion record was taken (Gray, 1971). In order to ensure that the animals allocated to the medial septal lesion group had sustained adequate, bilateral loss of hippocampal theta, allocation was made dependent on the extent of theta loss in whichever hemisphere showed the greatest retention of hippocampal theta. The rating system is thus conservative: rats could not be included unless their theta loss on the side least affected was sufficient; total theta loss on the side most affected was not considered to compensate for an inadequate loss contralaterally. Conversely, in allocating animals to the lateral septal (LS) group in which retention of theta was a requirement, acceptance depended upon there being adequate retention of theta in the hemisphere most seriously affected by the lesion; again, this procedure is conservative in that all animals with serious theta loss, even if only unilaterally, would be excluded.

Of the 12 animals implanted for medial septal lesions, 2 were excluded as showing too little prelesion theta and 4 because there was too little loss of theta after the lesion. The remaining 6 animals were acceptable on both electrophysiological and histological criteria, and they made up the final medial septal (MS) group. Of the 17 animals implanted for lateral septal lesions, 4 were excluded as showing too little prelesion theta and 7 were excluded because there was too much loss of theta after the lesion. Of the remaining 6 rats, one was excluded on histological grounds: the lesion extended too far in a rostral direction, was unilateral, and had slightly damaged the corpus callosum. The 6 animals that showed too little prelesion theta to be assigned to either lesion condition ( 2 with a medial septal electrode, 4 with lateral septal electrodes; see above) were assigned to the operated control (OC) group; one of these died before completion of behavioral testing, so the data for only the remaining 5 animals are reported. Thus, the final group sizes were: MS, 6; LS, 5; and OC, 5 .

\section{Behavioral Procedures}

The subjects were assigned randomly to one of three identical Campden Instruments CI $\mathbf{4 6 0}$ Skinner boxes, each equipped with a single lever and enclosed in a sound-attenuating outer chamber. The reinforcers were single 45-mg Campden Instruments pellets. The experiments were controlled by a NOVA 800 computer programmed in ACT-N (Millenson, 1971). After 1 day of magazine training, the subjects were given continuous reinforcement for 7 days; sessions terminated after $30 \mathrm{~min}$ or after 90 pellets had been delivered, whichever came sooner. The subjects were then shifted to DRL $20 \mathrm{sec}$. Session length was $30 \mathrm{~min}$, during which time the houselight and light above the lever were continuously on. The subjects were run in squads of three, one from each experimental condition. Data recorded were total numbers of responses and reinforcements per session and the number of responses in successive 5-sec bins (up to $30 \mathrm{sec}$ ) after each response (separately for rewarded and nonrewarded responses). All subjects were run on the DRL-20 schedule every day for 80 days. The data were analyzed in 16 consecutive blocks of 5 days.

\section{RESULTS}

Photographs of the lesions sustained by one MS and one LS animal are presented in Figure 1; Figure 2
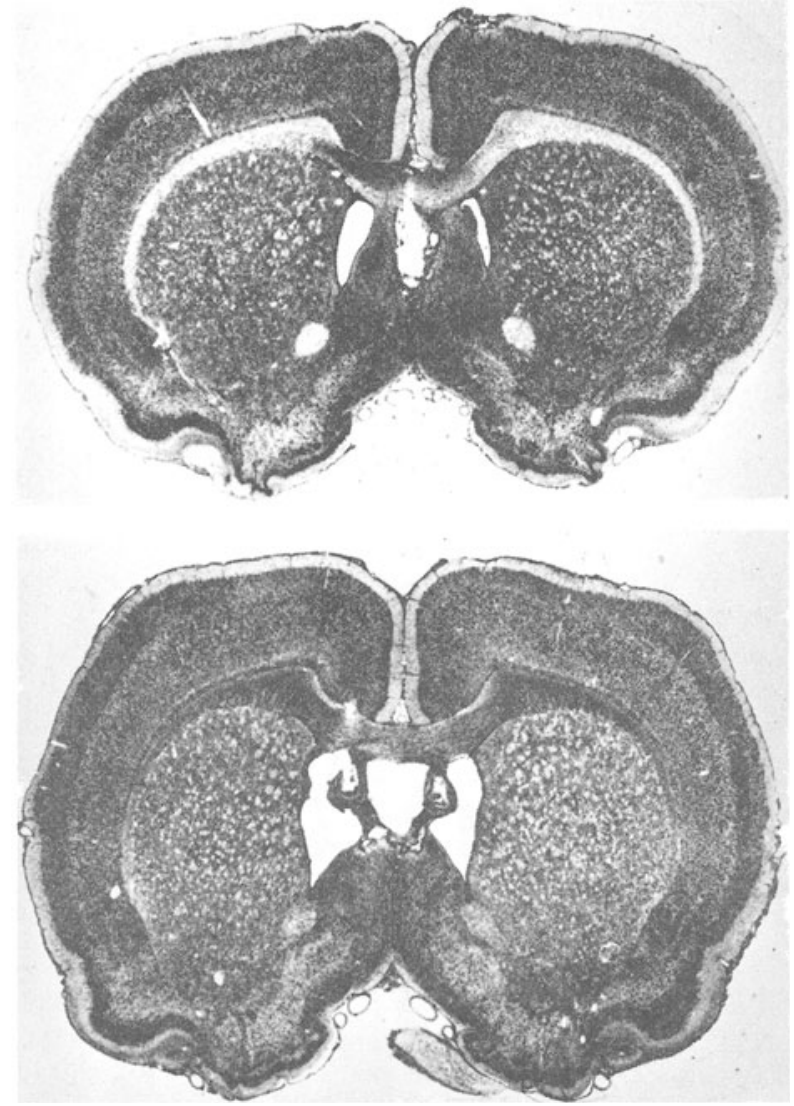

Figure 1. Photographs of coronal sections through the site of maximal damage in one animal with a medial septal lesion (above) and one with a lateral septal lesion (below). Sections $(30 \mu)$ stained with cresyl violet.

Whandwhw

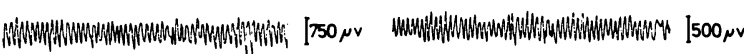

a

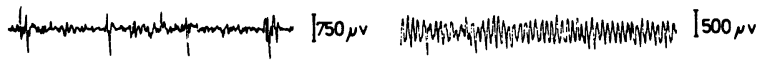

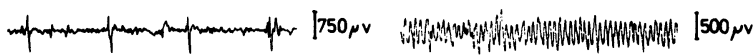

Figure 2. Hippocampal electrical activity from the left (upper trace) and right (lower trace) hemispheres before (A) and after (a) a medial septal lesion and before (B) and after (b) a lateral septal lesion. Time-marker, 1 sec; bar-markers show amplitude calibration. Filter settings: 1 and $75 \mathrm{~Hz}$. Histology for the same two rats is shown in Figure 1.

shows pre- and postlesion recordings of the same two animals. Of the six animals in the MS group, five (of which the subject illustrated is one) showed a 
postlesion reduction in theta amplitude (Rawlins et al., 1979) of more than $75 \%$ and the remaining subject, a reduction of more than $50 \%$. Of the five LS animals, three (including the subject illustrated) showed a postlesion reduction in theta amplitude of less than $25 \%$ and two, a reduction of less than $50 \%$. The lesions illustrated in Figure 1 were typical for the two groups. The MS lesions varied little rostrocaudally, although they did not always extend so far caudal as in the animal shown in Figure 1; ventrally, they often extended a little deeper than the case illustrated. LS lesions never extended more ventrally than the case shown in Figure 1.

There were no significant differences in response rate at the end of the initial period of continuous reinforcement. Figure 3 shows cumulative records for one session for each subject during Block 14 (Days 6670). Figure 4 shows percentage efficiency (rewards/ responses $\times 100$ ) for each lesion group at three different times during training. During Block 1 , the two lesion groups did not differ from each other and both were inferior to the operated controls (OC vs. MS, $U=4, p<.05$; OC vs. LS, $U=2, p<.02)$. By Block 2, these differences had disappeared, but they had reappeared by Block 14 (OC vs. MS, $U=2, p<$ .005 ; OC vs. LS, $U=3, p<.05$ ); the MS and LS groups continued to be indistinguishable.

Performance at the same three times during training is represented in Figure 5 in the form of "interresponse times per opportunity" (IRT/Opp). This metric describes the conditional probability of responding when an animal responds randomly in time (Anger, 1956). It can be seen that, by the end of training, all groups showed a clear timing curve, since the highest probability of responding occurred in the $20-25$-sec bin both after a previously rewarded response and after a previously nonrewarded response (Figure 5). Analysis of variance of the IRT/ Opp data over all 16 blocks confirmed this impres-

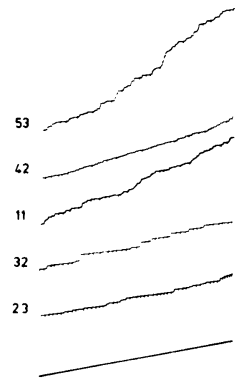

OPERATED CONTROLS

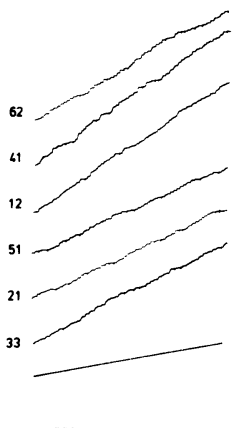

MEdial SEPTAL Lesions

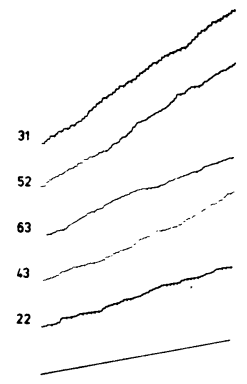

Lateral septal lesions
Figure 3. Cumulative records of barpresses during one session in Block 14 for every animal; numbers to the left identify individual rats. Responding at maximal efficiency would produce the gradient drawn at the bottom of each column. Downward defections indicate delivery of reinforcement. Note that Subject 51 was the medial septal rat and Subject 52 the lateral septal rat whose histology and hippocampal activity are presented in Figures 1 and 2.

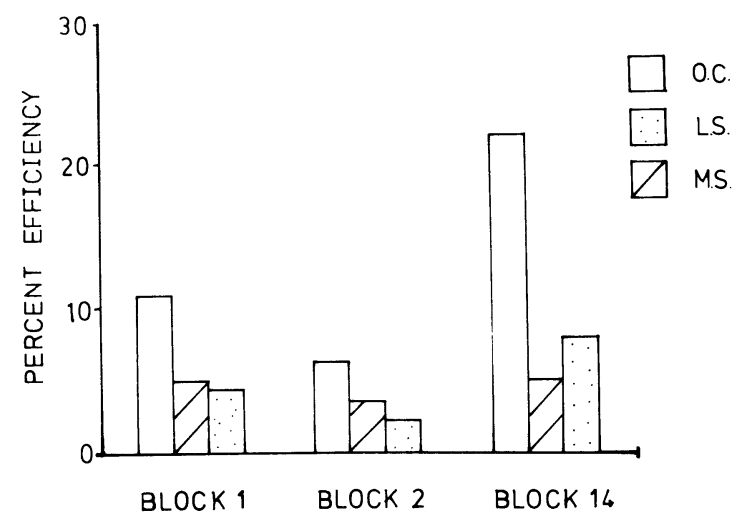

Figure 4. Percent efficiency (calculated as rewards/responses $\times 100)$ for each lesion group at three different times during training: Sessions 1-5 (Block 1), 6-10 (Block 2), and 66-70 (Block 14). OC, operated controls; LS, lateral septal lesions; MS, medial septal lesions.

sion: the main effect of bins was highly significant both postreward $(\mathrm{F}=81.5)$ and postnonreward $[\mathrm{F}(5$, $1040)=269.9, \mathrm{p}<.001$ ]; $\mathrm{t}$ tests showed that, in both cases, the probability of responding in the 20-25-sec bin was significantly higher than in any other bin.

The analysis revealed, in addition, significant main effects of lesion [postreward, $F(2,13)=5.34, p<.025$; postnonreward, $\mathrm{F}=10.52, \mathrm{p}<.01]$ and interactions between bins and lesion [postreward, $F(10,1040)=$ 7.3, $\mathrm{p}<.001$; postnonreward, $\mathrm{F}=13.02, \mathrm{p}<.001]$. None of these effects was attributable to differences between the MS and LS groups. Both of these groups had higher overall probabilities of responding than the controls: postreward means $=0.36,0.44$, and $0.43 \pm 0.026$ for the OC, MS, and LS groups, respectively; postnonreward means $=0.47,0.59$, and $0.59 \pm 0.030$. The bins $\times$ lesion interaction in the postreward data (as analyzed by $t$ test) was due to a significantly higher MS and LS response probability, relative to controls, in the 5-10- and 10-15-sec bins and a significantly lower LS response probability in the 25-30-sec bin. In the postnonreward data, both MS and LS rats were significantly more likely to respond than controls in all bins except the 0-5sec bin.

A final point to note is the clear difference between the postreward and postnonreward data with respect to the first bin (0-5-sec). In Block 14, for example, this bin had the lowest response probability in the postreward data but the highest of the first three bins in the postnonreward data (Figure 5). In neither set of data were there any differences between the three lesion conditions in this first bin.

\section{DISCUSSION}

Animals with both medial septal and lateral septal lesions showed a clear timing curve (Figure 5); thus, the capacity to estimate the passage of time is not 

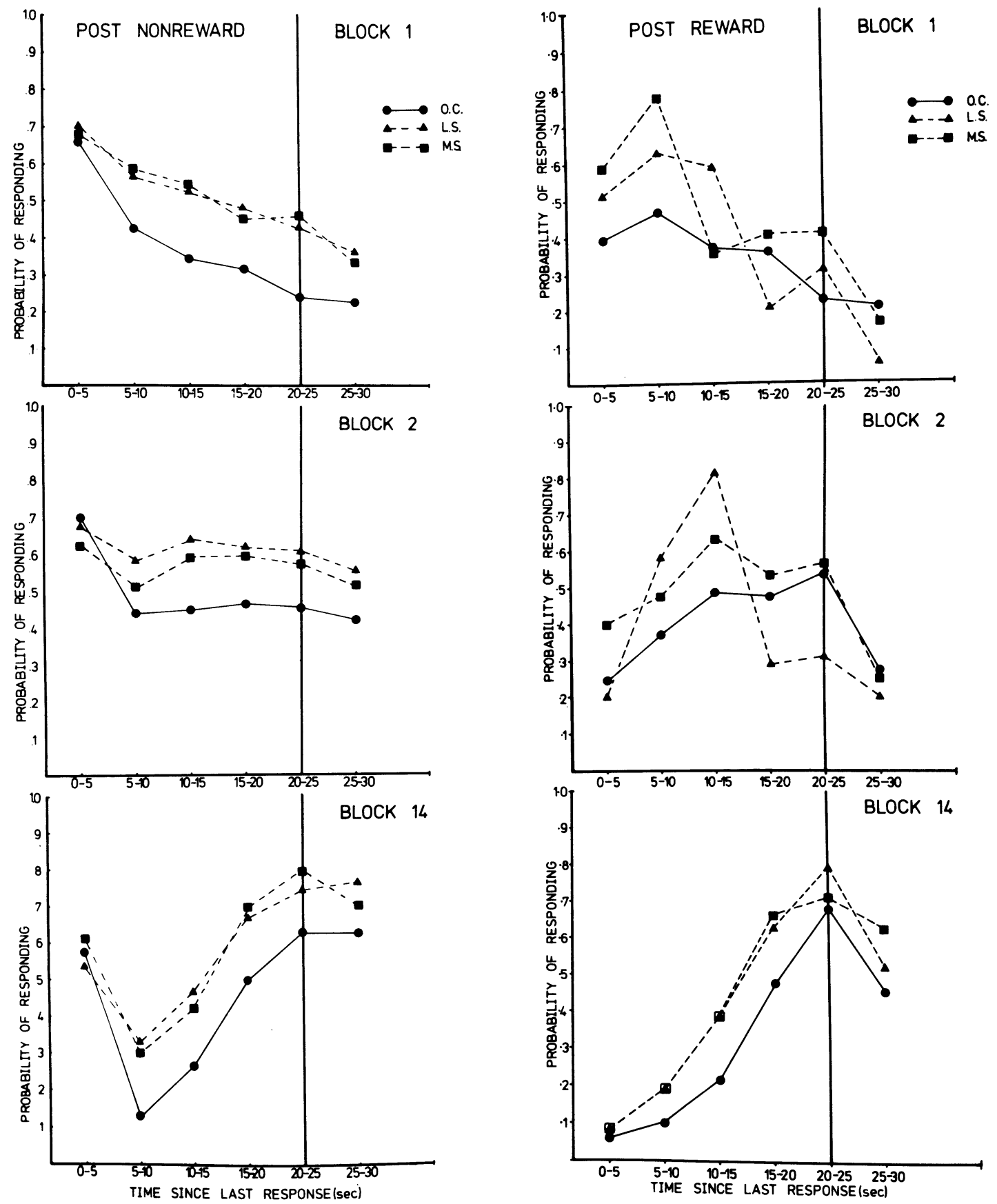

Figure 5. Plots of IRT/Opp (interresponse times per opportunity: Auger, 1956) for the three lesion groups as a function of blocks (1, 2, and 14 from top down) divided into responding following reward (right-hand column) and following nonreward (left-hand column). The ordinate shows the probability of responding in a particular time bin (abscissa) calculated as a proportion of responses in that and all longer time bins. Abbreviations as in Figure 4.

severely disrupted by these lesions. Nonetheless, both lesion groups were markedly impaired in their overall DRL performance. With the exception of the first bin $(0-5-\mathrm{sec})$, when, as our data confirm, behavior appears to be controlled by special features of the immediately preceding reward or nonreward (Carter \& Bruno, 1968; MacDougall, Van Hoesen, \& Mitchell,
1969), the animals with lesions consistently responded more than controls. This pattern of responding is consistent with the view that septal animals are impaired in their ability to inhibit behavior (McCleary, 1966).

The prediction, derived from Feldon and Gray's $(1979 a, 1979 b)$ findings using the partial reinforce- 
ment paradigm (see the introduction), that the impairment in DRL performance produced by total septal lesions would be reproduced by medial but not lateral septal lesions is clearly disconfirmed. Medial septal lesions had the predicted effect; but the behavior of the animals with lateral septal lesions was indistinguishable from that of the MS group. These findings confirm a previous report by Agnew and Meyer (1969). In that experiment, however, the medial and lateral septal lesions were larger than ours and overlapped one another; furthermore, the difference between the two lesions was not verified electrophysiologically, as it was in the experiment reported here.

A number of different features of the behavioral syndrome produced by total septal lesions may reasonably be attributed to a deficit in behavioral inhibition, including the DRL impairment (see above), impairment in punishment-produced or conditioned suppression, and increased resistance to extinction (Gray, 1982; McCleary, 1966). However, the data reported here, coupled with other findings from our laboratory, make it difficult to suppose that these three aspects of the septal syndrome all reflect the same underlying deficit. Using identical procedures to produce and verify medial and lateral septal lesions, we have shown that increased resistance to extinction is produced by medial, but not lateral, septal lesions (Feldon \& Gray, 1979a, 1979b); that reduced suppression of responses by punishment or stimuli associated with shock is produced by lateral, but not medial, septal lesions (Feldon, Rawlins, \& Gray, 1982a; see also Grossman, 1978; McGowan, Hankins, \& Garcia, 1972; Poplawsky \& Hoffman, 1979); and that the DRL deficit is produced equally by both kinds of lesion (present experiment).

\section{REFERENCE NOTE}

1. Feldon, J., Rawlins, J. N. P., \& Gray, J. A. The effects of fornix-fimbria section on the partial reinforcement extinction effect. Manuscript in preparation, 1983.

\section{REFERENCES}

Agnew, R. L., \& Meyer, M. E. Response inhibition of rats with medial and lateral septal lesions. Psychonomic Science, 1969, 16, 231-233.

Amsel, A. Frustrative nonreward in partial reinforcement and discrimination learning: Some recent history and a theoretical extension. Psychological Review, 1962, 69, 306-328.

ANGER, D. The dependence of interresponse times upon the relative reinforcement of different interresponse times. Journal of Experimental Psychology, 1956, 52, 145-161.

Burkett, E. E., \& Bunnell, B. N. Septal lesions and the retention of DRL performance in the rat. Journal of Comparative and Physiological Psychology, 1966, 62, 468-471.

Carter, D. E., \& Bruno, L. J. J. On the discriminative function of the reinforcing stimulus. Psychonomic Science, 1968, 11, 21-22.

Donovick, P. J. Effects of localized septal lesions on hippo- campal EEG activity and behavior in rats. Journal of Comparative and Physiological Psychology, 1968, 66, 569-578.

Ellen, P., Wilson, A. S., \& Powell, E. W. Septal inhibition and timing behavior in the rat. Experimental Neurology, 1964, 10, 120-132.

Elliott, K., \& Whelan, J. (Eds.), Functions of the septohippocampal system. Ciba Foundation Symposium 58 (New Series). Amsterdam: Elsevier, 1978.

Feldon, J., \& Gray, J. A. Effects of medial and lateral septal lesions on the partial reinforcement extinction effect at one trial a day. Quarterly Journal of Experimental Psychology, $1979,31,653-674$. (a)

Feldon, J., \& Gray, J. A. Effects of medial and lateral septal lesions on the partial reinforcement extinction effect at short inter-trial intervals. Quarterly Journal of Experimental Psychology, 1979, 31, 675-690. (b)

Feldon, J., \& Gray, J. A. The partial reinforcement extinction effect: Influence of chlordiazepoxide in septal-lesioned rats. Psychopharmacology, 1981, 74, 280-289.

Feldon, J., Rawlins, J. N. P., \& Gray, J. A. Effects of lateral and medial septal lesions on response suppression mediated by response-contingent and response-independent shock. Physiological Psychology, 1982, 10, 145-152. (a)

Feldon, J., Rawlins, J. N. P., \& Gray, J. A. Discrimination of response-contingent and response-independent shock by rats: Effects of medial and lateral septal lesions and chlordiazepoxide. Behavioral and Neural Biology, 1982, 35, 121135. (b)

GRAY, J. A. Sodium amobarbital, the hippocampal theta rhythm and the partial reinforcement extinction effect. Psychological Review, 1970, 77, 465-480.

Gray, J. A. Medial septal lesions, hippocampal theta rhythm and the control of vibrissal movement in the freely moving rat. Electroencephalography and Clinical Neurophysiology, 1971, 30, 189-197.

Gray, J. A. Precis of Gray's The Neuropsychology of anxiety: An enquiry into the functions of the septo-hippocampal system. Behavioral and Brain Sciences, 1982, 5, 469-484.

Gray, J. A., Feldon, J., Rawlins, J. N. P., Owen, S., \& McNaughton, N. The role of the septo-hippocampal system and its noradrenergic afferents in behavioural responses to nonreward. In K. Elliott \& J. Whelan (Eds.), Functions of the septohippocampal system. Ciba Foundation Symposium 58 (New Series). Amsterdam: Elsevier, 1978.

Gray, J. A., \& McNaughton, N. Comparison between the behavioural effects of septal and hippocampal lesions: A review. Neuroscience and Biobehavioral Reviews, 1983, 7, 119-188.

Grossman, S. P. An experimental 'dissection' of the septal syndrome. In K. Elliott \& J. Whelan (Eds.), Functions of the septohippocampal system. Ciba Foundation Symposium 58 (New Series). Amsterdam: Elsevier, 1978.

Henke, P. G. Persistence of runway performance after septal lesions in rats. Journal of Comparative and Physiological Psychology, 1974, 86, 760-767.

Henke, P. G. Dissociation of the frustration effect and the partial reinforcement extinction effect after limbic lesions in rats. Journal of Comparative and Physiological Psychology, 1977, 91, 1032-1038.

James, D. T., McNaughton, N., Rawlins, J. N. P., Feldon, J., \& GrAY, J. A. Septal driving of hippocampal theta rhythm as a function of frequency in the free-moving male rat. Neuroscience, 1977, 2, 1007-1017.

Konig, J. F. R., \& Klippel, R. A. The rat brain. Baltimore: Williams \& Wilkins, 1963.

Krayniak, P. F., Weiner, S., \& Siegel, A. An analysis of the efferent connections of the septal area in the cat. Brain Research, 1980, 189, 15-29.

McCleary, R. A. Response-modulating functions of the limbic system: Initiation and suppression. In E. Stellar \& J. M. Sprague (Eds.), Progress in physiological psychology (Vol. 1). New York: Academic Press, 1966. 
McDougall, J. M., Van Hoesen, G. W., \& Mitchell, J. C. Development of post $S^{r}$ and post non $S^{r}$ DRL performance and its retention following septal lesions in rats. Psychonomic Science, 1969, 16, 45-56.

McGowan, B. K., Hankins, W. G., \& Garcia, J. Limbic lesions and control of the internal and external environment. Behavioral Biology, 1972, 7, 841-852.

Millenson, J. R. A programming language for on-line control of psychological experiments. Behavioral Science, 1971, 16, 248-256.

O'KeEFE, J., \& NADEL, L. The hippocampus as a cognitive map. Oxford: Oxford University Press, 1978.

Pellegrino, L. J., \& ClapP, B. R. Limbic lesions and externally cued DRL performance. Physiology \& Behavior, 1971, 7, 863-868.

Petsche, H., Stumpf, Ch., \& Gogolak, G. The significance of the rabbit's septum as a relay station between the midbrain and the hippocampus. I. The control of hippocampus arousal activity by the septum cells. Electroencephalography and Clinical Neurophysiology, 1962, 14, 202-211.
Poplawsky, A., \& Hoffman, S. L. Effects of lateral, medial or complete septal lesions on response suppression. Physiology \& Behavior, 1979, 22, 679-682.

Rawlins, J. N. P., Feldon, J., \& Gray, J. A. Septo-hippocampal connections and the hippocampal theta rhythm. Experimental Brain Research, 1979, 37, 49-63.

Rawlins, J. N. P., Feldon, J., \& Gray, J. A. The effects of hippocampectomy and of fimbria section upon the partial reinforcement extinction effect in rats. Experimental Brain Research, 1980, 38, 273-283.

Swanson, L. W. The anatomical organization of septohippocampal projections. In K. Elliott \& J. Whelan (Eds.), Functions of the septo-hippocampal system. Ciba Foundation Symposium 58 (New Series). Amsterdam: Elsevier, 1978.

Swanson, L. W., \& Cowan, W. M. The connections of the septal region in the rat. Journal of Comparative Neurology, 1979, 186, 621-656.

(Manuscript received March 22, 1983;

revision accepted for publication September 13, 1983.) 